\title{
MACHINE-LEARNING TOOL FOR HUMAN FACTORS EVALUATION - APPLICATION TO LION AIR BOEING 737-8 MAX ACCIDENT
}

\author{
C. Morais ${ }^{1}$, K. Yung ${ }^{2}$, and E. Patelli ${ }^{1}$ \\ ${ }^{1}$ Institute for Risk and Uncertainty, University of Liverpool \\ Chadwick Building, Peach Street, Liverpool, L69 7ZF, United Kingdom \\ e-mail: \{caroline.morais, edoardo.patelli\}@ liverpool.ac.uk \\ ${ }^{2}$ Faculty of Applied Science \& Engineering, University of Toronto \\ 35 St. George Street, Room 157, Toronto, ON M5S 1A4, Canada \\ e-mail: kalai.yung@mail.utoronto.ca
}

\begin{abstract}
The capability of learning from accidents as quickly as possible allows preventing repeated mistakes to happen. This has been shown by the small time interval between two accidents with the same aircraft model: the Boeing 737-8 MAX. However, learning from major accidents and subsequently update the developed accident models has been proved to be a cumbersome process. This is because safety specialists use to take a long period of time to read and digest the information, as the accident reports are usually very detailed, long and sometimes with a difficult language and structure.

A strategy to automatically extract relevant information from report accidents and update model parameters is investigated. A machine-learning tool has been developed and trained on previous expert opinion on several accident reports. The intention is that for each new accident report that is issued, the machine can quickly identify the more relevant features in secondsinstead of waiting for some days for the expert opinion. This way, the model can be more quickly and dynamically updated. An application to the preliminary accident report of the 2018 Lion Air accident is provided to show the feasibility of the machine-learning proposed approach.
\end{abstract}

Keywords: Bayesian network updating, accident reports, Uncertainty Quantification, machine-learning, Boeing 737-8 MAX. 


\section{INTRODUCTION}

The industry should learn from past accidents to design and manage safer industrial installations, which is described by the 'learning from incidents' concept. There are some industrial recommended practices on how companies should use this concept [1] and research on how they are actually using it [2] or how it could be used [3]-[4]. The most acknowledged practice is the risk assessment, where a multi-disciplinary team revise the design according to information about past accidents, components reliability and human reliability.

Comprehensive risk assessments include human, organizational and technological factors [5], where human error probability is the likelihood of an individual to initiate or trigger a sequence of events that can lead to an accident. However, human behaviour is highly variable and depends not only on the individual but also on the organizational and technological factors all of them sources of aleatoric and epistemic uncertainties. To obtain these probabilities optimizing the 'learning from incidents' concept, Morais et al. have developed an approach that uses human factors data from major accidents and a probabilistic tool that accommodates those uncertainties [6]. Bayesian networks were chosen to model human errors due to the possibility of updating the model and its outputs with new evidence for each new accident report that is issued [7]. Reading an accident report and extracting the necessary information required to update the probabilities of the human errors require significant efforts and the availability of a risk specialist [8], resources that are not always available.

In the paper, a machine learning tool based on text recognition and supporting vector machine is proposed to automatically extract relevant information from accident reports. Previous works have used machine-learning to classify textual narratives for aviation and railway into defined (taxonomy) or dynamic (ontology) categories [9]-[10]. The main differences is that they have used a tanomy/ontology not entirely relevant for the human error model, and they have used voluntarily submitted reports, where the model needed inputs from investigation reports.

The proposed procedure also allows creating a "virtual risk expert" trained on using predefined taxometry. The "virtual expert" is than able to process accident reports and extract relevant information in real-time. The proposed methodology is applied to analyse the accident report of the 2018 Lion Air accident [11] is provided to show the feasibility of the machinelearning proposed approach. The approach proposed allows also to understand how the same type of error is perceived and classified in different sectors.

\section{METHODOLOGY}

\subsection{A Bayesian network to predict human error in complex industries}

To build a model of human error in complex industries the Bayesian networks proposed by by some of the authors has been used [6]. Bayesian Networks are a probabilistic tool that can be presented in the form of a directed acyclic graph made of nodes (variables) connected by links. The open source Bayesian Network toolbox [12] implanted in OpenCossan [12]-[14] has been used to analyse and evaluate the developed model. The probability values denoting the degree of dependency within the nodes are stored in a conditional probability table, thus each

state of the child is provided given each of the states of the parents. The product of all the conditional and unconditional probabilities specified in the network is governed by the chain rule for Bayesian networks [15].

In [6], to build the structure of the Bayesian network the authors have used the dependency among the variables proposed in [8]. This arrangement of parents and children nodes connected 
by links allows predictive and diagnostic calculations. Therefore, not only human error probabilities can be predicted but also the factors that contribute to those error can also be further investigated. A simplified version of the originally developed model is shown in Figure 1 where the nodes are related to the CREAM taxonomy (Cognitive reliability and error analysis method) for human errors and organisational, technological and individual factors [16]. The CREAM's features adopted in the model are shown in Table 1. The probability values for each node are based on MATA-D (Multiattribute Technological Accidents Dataset), a dataset created by experts (risk analysts) [3], after reading accident reports and classifying them as boolean values ( 0 for absent, 1 for present) according to the features described in Table 1.

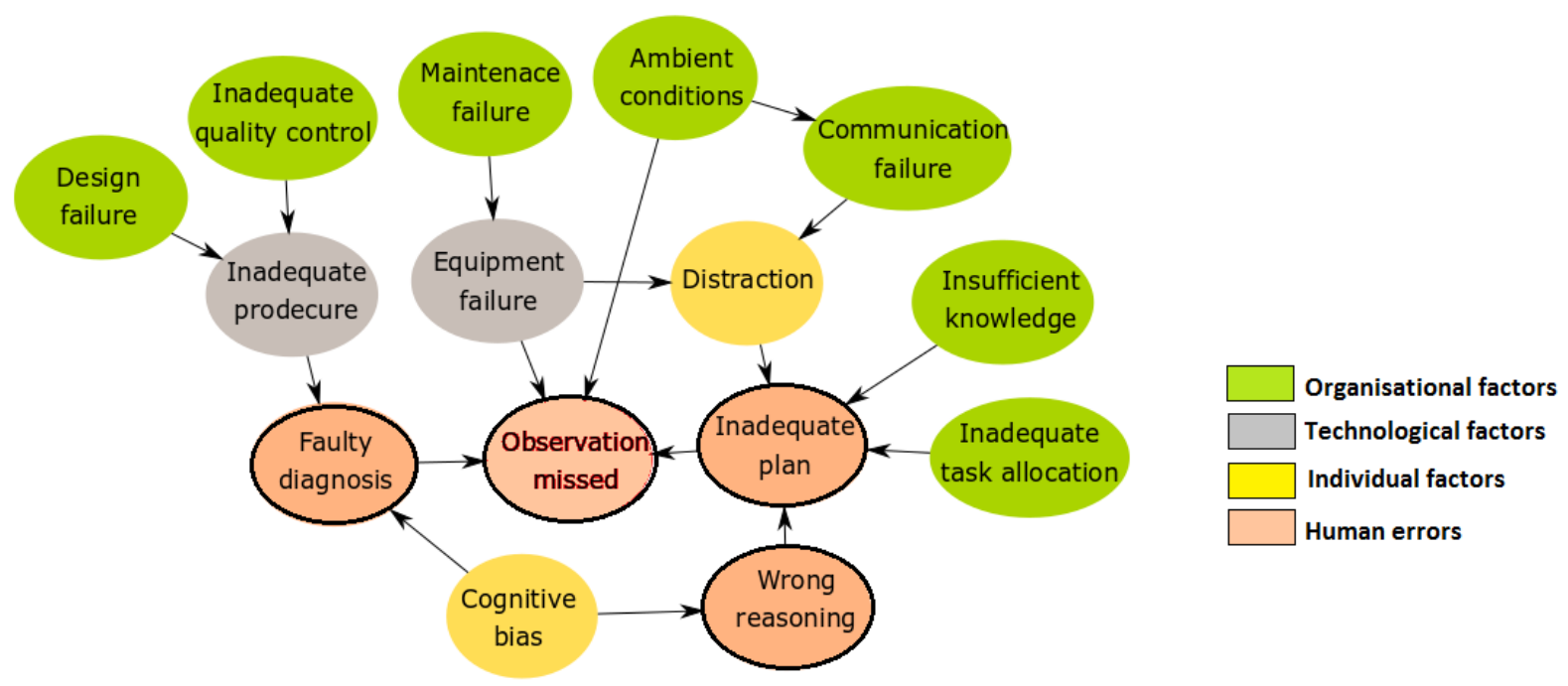

Figure 1 - Simplified representation of the model for Human error derived from [6].

Table 1. CREAM features of human factors adoped in the proposed Human error model.

\begin{tabular}{llll}
\hline Organisational Factors & Technological Factors & Individual factors & Human Errors \\
\hline Communication failure & Equipment failure & Permanent related & Cognitive Errors \\
\hline Missing information & Software fault & Functional impairment & Observation missed \\
\hline Maintenance failure & Inadequate procedure & Cognitive style & False Observation \\
\hline Inadequate quality control & Access limitations & Cognitive bias & Wrong Identification \\
\hline Management problem & Ambiguous information & Temporary & Faulty diagnosis \\
\hline Design failure & Incomplete information & Temporary related & Wrong reasoning \\
\hline Inadequate task allocation & Access problems & Memory failure & Decision error \\
\hline Social pressure & Mislabelling & Fear & Delayed interpretation \\
\hline Insufficient skills & & Distraction & Incorrect prediction \\
\hline Insufficient knowledge & & Fatigue & Inadequate plan \\
\hline Adverse ambient conditions & & Performance Variability & Priority error \\
\hline Excessive demand & & Inattention & Execution Errors \\
\hline Inadequate work place layout & & Physiological stress & Wrong time \\
\hline Irregular working hours & & Psychological stress & Wrong type \\
\hline & & & Wrong Object \\
\hline
\end{tabular}




\subsection{Updating Bayesian network probabilities via a machine-learning approach}

In an ideal situation, the Bayesian network is updated every time a new accident report is released. Hence, human error probabilities are updated with changes in organizational and technological factors. However, each report has two hundred pages on average, and the reading and classification into a taxonomy is a time-consuming task. Therefore, it was idealised that a trained machine could help on this cumbersome task. A semi-supervised training algorithm could automatically classify the report supporting the analysis of an analyst.

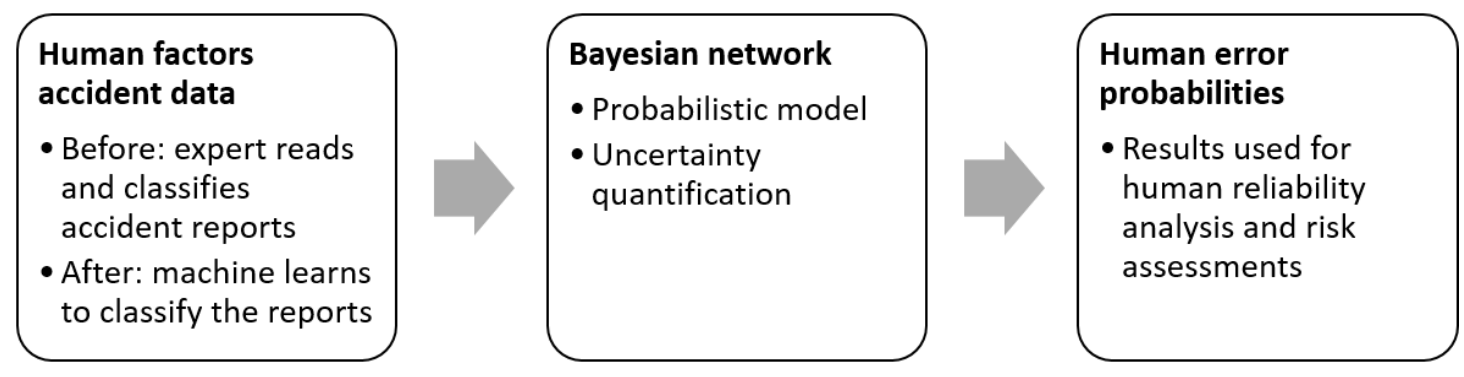

Figure 2 - Conceptual approach to update a human error probability model.

A semi-supervised approach is proposed to analyse accident report and update human error probabilities in the proposed model. The concept of the machine-learning approach is summarised in Figure 2. For this study, the Matlab text analytics toolbox based on the bag of words model [17] is used for extracting text strings from PDF files and preparing the data for the machine-learning algorithm. The Matlab statistics and the machine-learning toolbox is used for transforming text inputs into binary classification adopting Support Vector Machine [18]. A brief background on the models for text selection and machine-learning is here provided.

A bag-of-words model is a way of extracting features from the text, representing it by the vocabulary of known words and a measure of their occurrence. However, it does not provide any information about the order or structure of words - the reason that it is called a "bag" of words. To apply it to a collection of documents, first the data is collected from the text files. Then a vocabulary is prepared by making a list of all the words in the text. To improve the results and save computational time and memory the model ignores case, punctuation, and other frequent words that do not contain relevant information, such as stop words (e.g. 'a', 'the', 'of'). To score the words in each document the presence of known words is marked as a boolean value ( 0 for absent, 1 for present). Thus, using the list of words previously prepared, the new document is analysed and converted into a binary vector. To extract the features from the documents, the ordering of the words is discarded [17].

The Support Vector Machine is the machine-learning algorithm used, popular due to the little need for adjustments. In the simplest case - when the data has exactly two classes - the Support Vector Machine classifies data by finding the "maximum-margin hyperplane" hyperplane that separates the data points of one class (type 1, represented in the Figure 3 as + ) from those of the other class (type -1, represented on Figure 3 as - ). Any hyperplane can be written as the set of points $\mathbf{x}$ satisfying:

$$
\mathbf{w} \cdot \mathbf{x}-\mathrm{b}=0 \quad \text { (Equation } 1)
$$

where $\mathbf{w}$ is the normal vector to the hyperplane. The parameter $b /\|\mathbf{w}\|$ determines the offset of the hyperplane from the origin along the normal vector $\mathbf{w}$ as shown in Figure 3 . The hyperplanes that defines the classes are can be described by the following equations: 


$$
\begin{aligned}
& \mathbf{w} \cdot \mathbf{x}-\mathrm{b}=1 \\
& \text { (Equation 2) } \\
& \mathbf{w} \cdot \mathbf{x}-b=-1 \\
& \text { (Equation 3) }
\end{aligned}
$$

The support vectors are the data points that are closest to the separating hyperplane; these points are on the boundary of the slab. As it is a supervised learning model the Support Vector Machine has to be trained first and then cross-validate the classifier. After that, the trained machine can be used to predict or classify new data. In addition, to obtain satisfactory predictive accuracy, various Support Vector Machine kernel functions can be used.

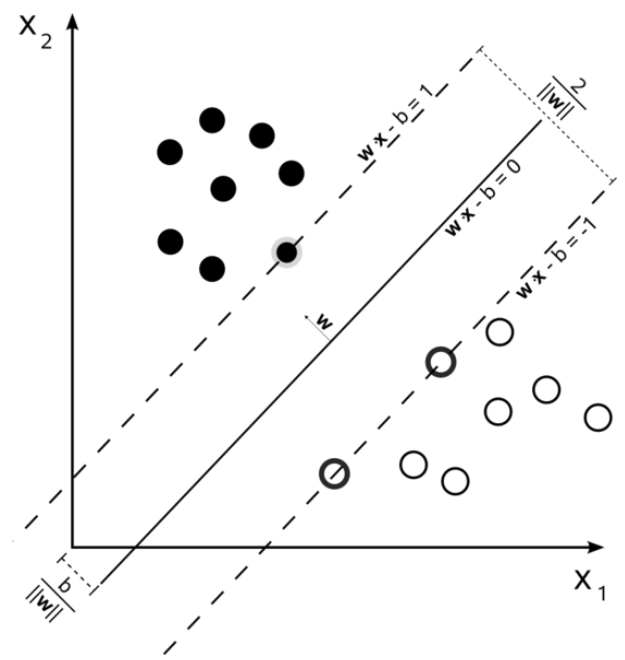

Figure 3 - Conceptual illustration of the support vector machine [19]

\subsection{Machine-learning tool overview}

The proposed machine-learning approach is trained using accident reports classified beforehand by experts according to CREAM taxonomy with the aim of being able to predict automatically the features of similar accident reports. A simplified workflow of the proposed approach is shown in Figure 4.

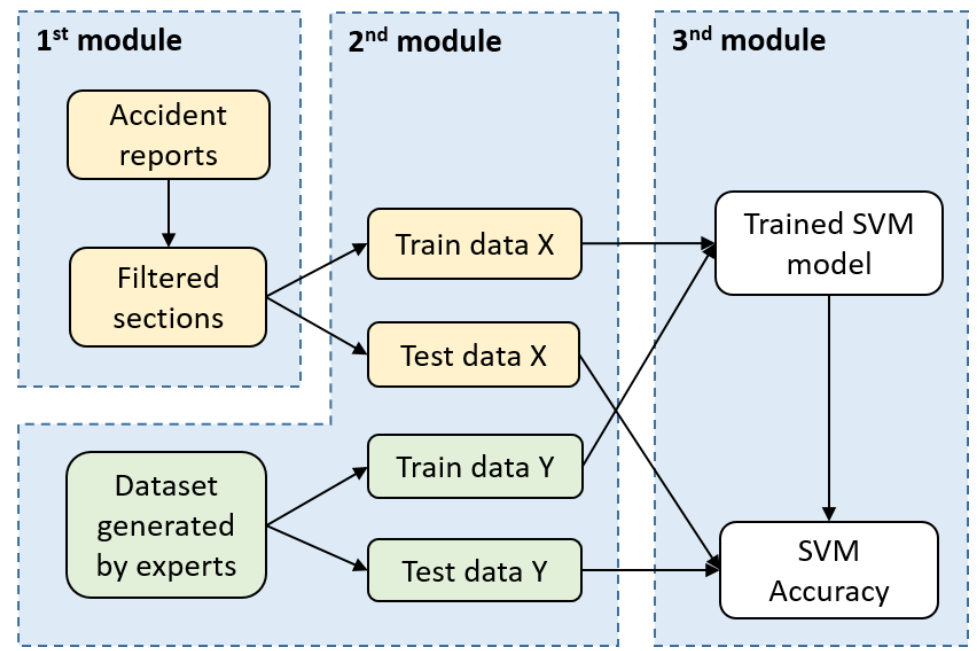

Figure 4 - Simplified workflow of the proposed approach

In the first module of the tool, accident investigation reports are used as inputs. In this study, the documents were in PDF (portable document format). It is important to use an Optical Character Recognition (OCR) software on accident reports that were shared as image files, in order 
to convert them in text files. After this pre-treatment, the accident reports are scanned and relevant sections are identified for the sake of efficiency. In the current implantation, the semisupervised approach selects the recommendation, lessons learned, and advice sections of the incident reports. Using a scoring system, the most likely starts and ends of the target sections are identified, and the sections' texts are sent to later code.

The second module of the tool aggregates the aforementioned section texts with the MATA$\mathrm{D}$ dataset information needed to begin machine learning. This dataset contains human factors features to be used as desired outputs for machine-learning. Using another scoring system, the tool takes each accident report's file name and finds the most likely corresponding entry in the MATA-D dataset (as each report listed in the dataset has a correspondent PDF file). This gives the machine-learning component the desired output (i.e. the correct categories) for each incident report. This module's output is a combination of selected section texts and the known human factors features of them.

In the third and last module, the machine-learning model based on support vector machine is trained and tested using the data input from the previous two modules. The section texts are converted into BagOfWords objects as X. The features extracted from the MATA-D data serves as the $\mathrm{Y}$. The module partitions the $\mathrm{X}$ and $\mathrm{Y}$ data into a training set (90\% of total) and a testing set (10\% of total). For each CREAM feature, an SVM model is trained using training X and Y sets, then it is tested using the testing $\mathrm{X}$ and $\mathrm{Y}$ sets. At the same time, run information is recorded and overall accuracy of all test sets in all categories is calculated.

\subsection{Accuracy of the machine-learning model created}

Each accident report was treated as a document, and the set of accident reports of one specific investigation body was treated as a corpus of documents, The current collection of reports comes from different organizations with considerably different formats and vocabularies. The formats range from a few concise pages in Chemical Safety Board reports to a 200-page letter to the US president on the BP oil spill. In this paper, two corpora used were: the US National Transportation Safety Board (NTSB), that investigates aviation accidents, and the U.S. Chemical Safety and Hazard Investigation Board (CSB), that investigates industrial chemical accidents.

If the machine-learning model is only trained with the NTSB reports, the overall accuracy of the test sets is approximately $85 \%$. If the model is trained with US Chemical Safety and Hazard Investigation Board reports, the accuracy is approximately $91 \%$. This is possibly due to the different number of training data for both corpus; the MATA-D dataset had classified 39 CSB reports and 13 NTSB reports, among a total of 238 accident reports from different industry sectors. However, $85 \%$ is considered an equally good result for the classification of narrative reports into a taxonomy, especially if considered that the inter-rater reliabilities within experts are considered acceptable if the label accuracy is above $70 \%$ [9].

\section{CASE-STUDY - 2018 ACCIDENT WITH BOEING 737 MAX 8 AIRCRAFT}

On 2018, an accident with a Lion Air plane has lead to 188 fatalities (two pilots, five flight attendants and 181 passengers) [11]. Five months later, in 2019, an Ethiopian Airlines plane has crashed minutes after take-off, killing all 157 people on board [20][20]. The fact that both planes were the same model, a Boeing 737-8 MAX, concerned civil society and safety regulators about the possible common flaws on all planes within the same model, resulting in all 387 Boeing 737 Max 8 planes grounded globally [21]. This illustrates the importance of learning from accidents before making informed decisions. 
For this research the preliminary accident report of the Lion Air Aircraft flight [11] has been tested with the newly developed and trained machine-learning tool, after training the machine with two different training sets: a set with only aviation accident investigation reports and a set with only with chemical industries accident investigation reports. All of the documents were previously classified by an expert within the CREAM human factors taxonomy as shown in Table 1.

\subsection{Major findings}

The results obtained by the machine after being trained by NTSB (aviation) and CSB (chemical) accident reports can be compared in Table 2 for human errors and Table 3 for the factors that might trigger them. The performance of the machine-learning based tool depends on the quality of the training data. For instance, if the machine learning tool is trained only with aviation reports, it classify four types of human errors against only one if it is trained by chemical accident reports. Also, among the three types of factors (organizational, technological and individual) that may trigger human errors, the machine results after being trained by aviation reports focus much more on individual factors than when trained with chemical reports - giving more weight on the human responsibility upon the system, than the system upon the human. This trend can be better identified in Figure 5, after joining the 53 features into their four highest levels. There are some possible reasons why the training provided by the chemical accident reports are more emphatic on organisational factors, but one is important to discuss: the results on the preliminary report of Lion Airlines flight accident might be describing more about the training corpus them about the actual report. This means that chemical industries might have much more organisational factors initiating accident events than in aviation. This is certainly true in the case of the 'maintenance failure' factor. It is possible that a maintenance error initiates an event on a flight, but in chemical industries the probability is much higher as maintenance tasks can be executed while the system operates. Accordingly, it is understandable why human errors and individual factors are much more explored in aviation accident reports than in chemical plants. In aviation, the investigation is focused in the cockpit, on the crew performance. On the other hand, it is not clear to which extent investigators are not digging more to the organisational and technological factors that are triggering the human errors of the crews.

Table 2 - Human errors identified

\begin{tabular}{|c|c|c|c|c|}
\hline & & & $\begin{array}{l}\text { Trained with aviation } \\
\text { reports (NTSB) }\end{array}$ & $\begin{array}{l}\text { Trained with chemical } \\
\text { accident reports (CSB) }\end{array}$ \\
\hline \multirow{14}{*}{$\begin{array}{c}\text { Human } \\
\text { errors }\end{array}$} & \multirow{4}{*}{ Execution } & 'Wrong Time' & Yes & Yes \\
\hline & & 'Wrong Type' & 0 & 0 \\
\hline & & 'Wrong Object' & 0 & 0 \\
\hline & & 'Wrong Place' & Yes & 0 \\
\hline & \multirow[t]{3}{*}{ Observation } & 'Observation Missed' & 0 & 0 \\
\hline & & 'False Observation' & 0 & 0 \\
\hline & & 'Wrong Identification' & Yes & 0 \\
\hline & \multirow[t]{5}{*}{ Interpretation } & 'Faulty diagnosis' & Yes & 0 \\
\hline & & 'Wrong reasoning' & 0 & 0 \\
\hline & & 'Decision error' & 0 & 0 \\
\hline & & $\begin{array}{l}\text { 'Delayed interpreta- } \\
\text { tion' }\end{array}$ & 0 & 0 \\
\hline & & 'Incorrect prediction' & 0 & 0 \\
\hline & \multirow[t]{2}{*}{ Planning } & 'Inadequate plan' & 0 & 0 \\
\hline & & 'Priority error' & 0 & 0 \\
\hline
\end{tabular}


Table 3 - Organizational, Technological and Individual factors that may trigger human errors

\begin{tabular}{|c|c|c|c|c|}
\hline & & & $\begin{array}{l}\text { Trained with avia- } \\
\text { tion reports }\end{array}$ & $\begin{array}{l}\text { Trained with chemical } \\
\text { accident reports }\end{array}$ \\
\hline \multirow{20}{*}{ 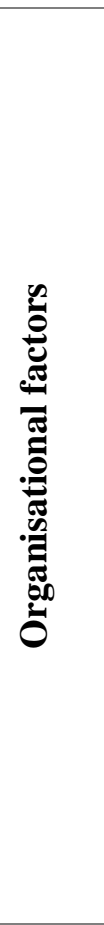 } & \multirow{2}{*}{$\begin{array}{c}\text { Communica- } \\
\text { tion }\end{array}$} & 'Communication failure' & Yes & 0 \\
\hline & & 'Missing information' & 0 & Yes \\
\hline & \multirow[t]{6}{*}{ Organisation } & 'Maintenance failure' & 0 & Yes \\
\hline & & 'Inadequate quality control' & Yes & Yes \\
\hline & & 'Management problem' & 0 & Yes \\
\hline & & 'Design failure' & 0 & Yes \\
\hline & & 'Inadequate task allocation' & 0 & Yes \\
\hline & & 'Social pressure' & 0 & 0 \\
\hline & \multirow[t]{2}{*}{ Training } & 'Insufficient skills' & 0 & Yes \\
\hline & & 'Insufficient knowledge' & 0 & 0 \\
\hline & \multirow{6}{*}{$\begin{array}{l}\text { Ambient Con- } \\
\text { ditions }\end{array}$} & 'Temperature' & 0 & 0 \\
\hline & & 'Sound' & 0 & 0 \\
\hline & & 'Humidity' & 0 & 0 \\
\hline & & 'Illumination' & 0 & 0 \\
\hline & & 'Other' & 0 & 0 \\
\hline & & 'Adverse ambient conditions' & 0 & 0 \\
\hline & \multirow{4}{*}{$\begin{array}{l}\text { Working } \\
\text { Conditions }\end{array}$} & 'Excessive demand' & 0 & 0 \\
\hline & & 'Inadequate work place layout' & 0 & 0 \\
\hline & & 'Inadequate team support' & 0 & 0 \\
\hline & & 'Irregular working hours' & 0 & 0 \\
\hline \multirow{8}{*}{ 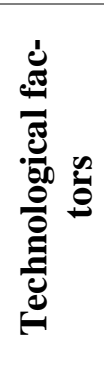 } & \multirow[t]{2}{*}{ Equipment } & 'Equipment failure' & 0 & Yes \\
\hline & & 'Software fault' & 0 & 0 \\
\hline & Procedures & 'Inadequate procedure' & Yes & 0 \\
\hline & \multirow{3}{*}{$\begin{array}{l}\text { Temporary } \\
\text { Interface }\end{array}$} & 'Access limitations' & 0 & 0 \\
\hline & & 'Ambiguous information' & 0 & 0 \\
\hline & & 'Incomplete information' & 0 & Yes \\
\hline & \multirow{2}{*}{$\begin{array}{l}\text { Permanent } \\
\text { Interface }\end{array}$} & 'Access problems' & 0 & 0 \\
\hline & & 'Mislabelling' & 0 & 0 \\
\hline \multirow{11}{*}{ 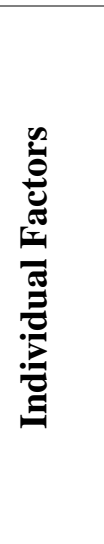 } & \multirow{8}{*}{$\begin{array}{c}\text { Temporary } \\
\text { Person } \\
\text { Related } \\
\text { Factors }\end{array}$} & 'Memory failure' & 0 & 0 \\
\hline & & 'Fear' & 0 & 0 \\
\hline & & 'Distraction' & Yes & Yes \\
\hline & & 'Fatigue' & Yes & 0 \\
\hline & & 'Performance Variability' & Yes & 0 \\
\hline & & 'Inattention' & Yes & 0 \\
\hline & & 'Physiological stress' & 0 & 0 \\
\hline & & 'Psychological stress' & 0 & 0 \\
\hline & \multirow{3}{*}{$\begin{array}{c}\text { Permanent } \\
\text { Person } \\
\text { Related } \\
\text { Factors }\end{array}$} & 'Functional impairment' & 0 & 0 \\
\hline & & 'Cognitive style' & 0 & 0 \\
\hline & & 'Cognitive bias' & Yes & 0 \\
\hline
\end{tabular}




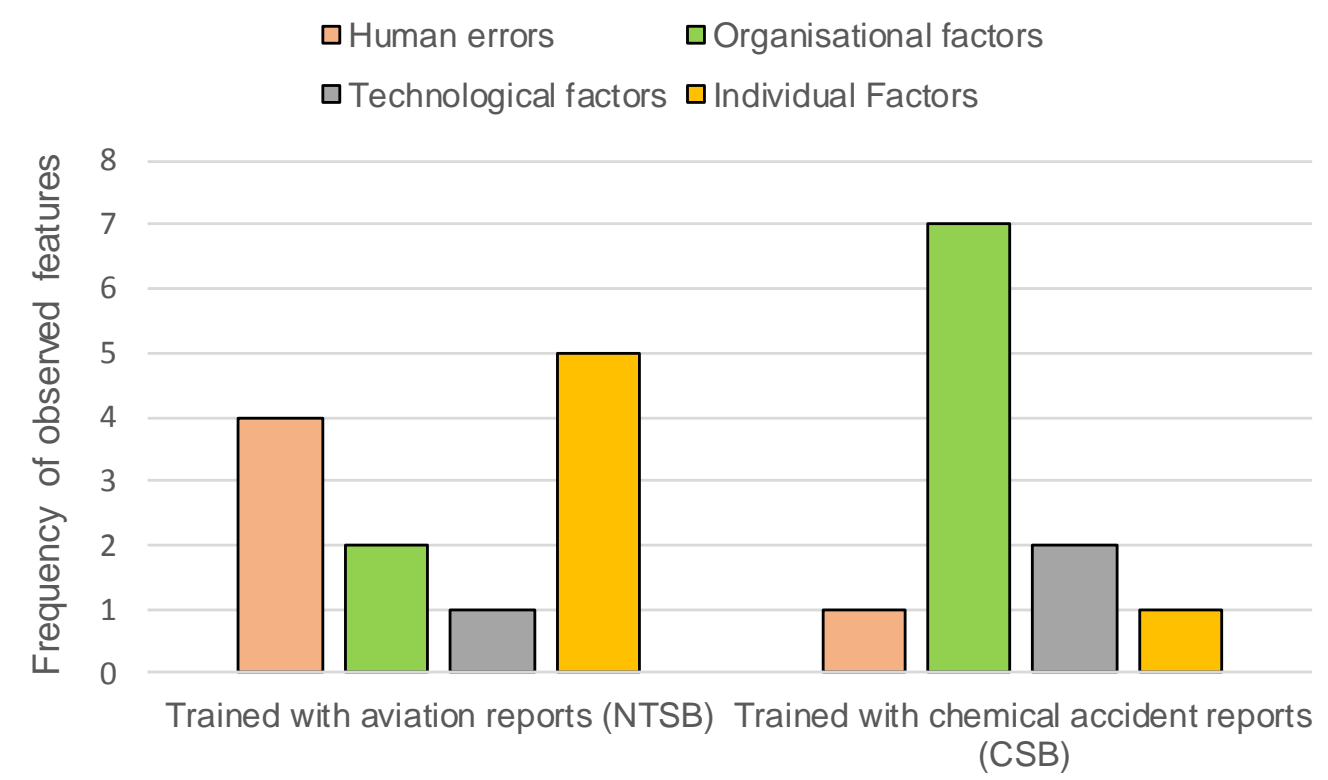

Figure 5 - Observed features after the machine is trained in different types of report

Another important aspect to be considered is the comparison of the present results to what is being communicated by the media and specialists in the area. Although recent news from media (e.g. [21]) and specialists opinion (e.g. [22]) accounts for the possible inadequacy of the software installed on the plane, the developed machine-learning tool has not identified the feature 'software fault' of the taxonomy. Some possible reasons for the lack of 'software fault' identification by the machine are described below:

- The document tested is a preliminary investigation report for the accident occurred to Lion Airlines (on 28 October 2018). There are few mentions to 'AoA' sensor but they do not state a definite problem with it, as illustrated by this sentence extracted from the report: "The investigation will perform several tests including the test of the 'AoA' sensor and the aircraft simulator exercises in the Boeing engineering simulator. The investigation has received the QAR data for flight for analysis". Thus, as the data was not yet evaluated and a final report was not issued, the software problem pointed by the media is not official - and it was possible to be perceived as one of the causes only after the accident occurred to Ethiopian Airlines (on 10 March 2019).

- The points stated about the sensors, on this preliminary report, use lots of acronyms or field-specific words possibly not yet related in other accident investigation reports. This could be tackled by training the machine also in the acronyms and specific words for each field, such as those reported in the Aviation Safety Reporting System (ASRS). This might also be improved by using a machine-learning strategy that accounting for the order of the words.

- The model and machine-learning tool developed has not yet achieved $100 \%$ accuracy. If it was, maybe could detect the software problem on the preliminary report. It is currently achieving $85 \%$ accuracy (if the machine is trained with aviation NTSB reports) and $91 \%$ accuracy (if trained with Chemical CSB reports). 


\section{CONCLUSIONS}

This study shows the feasibility of implementing a machine-learning tool to update the Bayesian network probabilities by scanning new reports without the necessity of the time consuming and expensive approach required by the traditional task. The proposed approach is based on text-recognition and text-classification, combined with support vector machine for classifying text according to predefined taxonomy to create a "virtual risk expert". This allows a real-time update of the model parameter available and it can be of fundamental importance to identify main causes of patterns across accidents.

The case study about the Boeing 737 MAX-8 plane accident has been presented showing that new evidence can be included in the Bayesian network proposed and new human error probabilities can be generated. The results of the analysis show that human factors are revealed when the model is trained using data from the chemical industry and not only from aviation, indicating the importance of cross-discipline knowledge transfer.

\section{ACKNOWLEDGEMENTS}

We would like to acknowledge the efforts of Jack Tully and Hao Xu, final year undergraduate students of the University of Liverpool that have contributed to improve and test the code. This research has been supported by ANP, the Brazilian Oil \& Gas Regulator, and by EPSRC under the grant EP/R020558/1.

\section{REFERENCES}

[1] Center for Chemical Process Safety (CCPS), 2010. Guidelines for Risk Based Process Safety. John Wiley \& Sons.

[2] Drupsteen, L., Groeneweg, J. and Zwetsloot, G.I., 2013. Critical steps in learning from incidents: using learning potential in the process from reporting an incident to accident prevention. International journal of occupational safety and ergonomics, 19(1), pp.63-77.

[3] Moura, R.; Beer, M.; Patelli, E.; Lewis, J. and Knoll, F., 2017. Learning from accidents: interactions between human factors, technology and organisations as a central element to validate risk studies. Safety Science, 99, pp.196-214. DOI: 10.1016/j.ssci.2017.05.001

[4] Moura, R.; Beer, M.; Patelli, E. \&amp; Lewis, J. 2017 Learning from major accidents: graphical representation and analysis of multi-attribute events to enhance risk communication. Safety Science, 99, 58-70 DOI: 10.1016/j.ssci.2017.03.005

[5] Zio, E., 2018. The future of risk assessment. Reliability Engineering \& System Safety, 177, pp.176-190.

[6] Morais, C., Moura, R., Beer, M., Patelli, E., 2019 (in press) Analysis and estimation of human errors from major accident investigation reports. ASCE-ASME Journal of Risk and Uncertainty in Engineering Systems, Part B: Mechanical Engineering.

[7] Estrada-Lugo H.D., Tolo S., de Angelis M. and Patelli, E. 2019 (in press) An inference method for bayesian networks with probability intervals ASCE-ASME Journal of Risk and Uncertainty in Engineering Systems Part B: Mechanical Engineering

[8] Moura, R., Beer, M., Patelli, E., Lewis, J. and Knoll, F., 2016. Learning from major accidents to improve system design. Safety science, 84, pp.37-45. DOI: 10.1016/j.ssci.2015.11.022 
[9] Robinson, S., 2018. Multi-label classification of contributing causal factors in self-reported safety narratives. Safety, 4(3), p.30.

[10] Van Gulijk, Coen; HUGHES, Peter; FIGUERES-ESTEBAN, Miguel. The potential of ontology for safety and risk analysis. In: Proceedings of ESREL 2016. CRC Press, 2016.

[11] Transportasi, K.N.K., 2018. Preliminary KNKT.18.10.35.04 Aircraft accident investigation report, PT. Lion Mentari Airlines, Boeing 737-8 (MAX). Ministry of Transportation, Indonesia, Report.

[12] Tolo, S., Patelli, E. and Beer, M. 2018, An open toolbox for the reduction, inference computation and sensitivity analysis of Credal Networks. Advances in Engineering Software, 115, 126-148. DOI: 10.1016/j.advengsoft.2017.09.003

[13] Patelli, E., 2016. COSSAN: a multidisciplinary software suite for uncertainty quantification and risk management. In Handbook of uncertainty quantification, pp.1-69. DOI: 10.1007/978-3-31911259-6_59-1

[14] Patelli, E.; George-Williams, H.; Sadeghi, J.; Rocchetta, R.; Broggi, M. and de Angelis, M. 2018, OpenCossan 2.0: an efficient computational toolbox for risk, reliability and resilience analysis Proceedings of the joint ICVRAM ISUMA UNCERTAINTIES conference, http://icvramisuma2018.org/cd/web/PDF/ICVRAMISUMA2018-0022.PDF

[15] Tolo, S. and Patelli, 2015, A computational tool for Bayesian network enanched with reliability methods, Proceedings of the 1st ECCOMAS Thematic Conference on Uncertainty Quantification in Computational Sciences and Engineering Crete Island, Greece, 25-27 May 2015, Eccomas Proceedia ID: 4316, 908-923

[16] Hollnagel, E., 1998. Cognitive reliability and error analysis method (CREAM). Elsevier.

[17] Brownlee, Jason Brownlee. "A Gentle Introduction to the Bag-of-Words Model." Machine Learning Mastery, https://machinelearningmastery.com/gentle-introduction-bag-words-model/, website visited on $1^{\text {st }}$ May 2019

[18] Malab documentation. Support Vector Machines for Binary Classification. https://www.mathworks.com/help/stats/support-vector-machines-for-binary-classification.html. Website visited on $1^{\text {st }}$ May 2019

[19] Wikipedia https://commons.wikimedia.org/wiki/File:Svm_max_sep_hyperplane_with_ margin.png.

[20] BBC News. https://www.bbc.co.uk/news/business-47523468. Website visited on 3rd April 2019

[21] BBC News. https://www.bbc.co.uk/news/business-48038026?intlink_from_url= https://www.bbc.co.uk/news/topics/c2g0x3qg9q1t/boeing-737-max-8\&link_location=live-reporting-story. Website visited on 25th April 2019.

[22] Gregory Travis Specialist opinion on Boeing 737 MAX-8 failures. IEEE Spectrum. 19 April 2019. Available at: https://spectrum.ieee.org/aerospace/aviation/how-the-boeing-737-max-disaster-looks-to-a-software-developer . 